\title{
REOLOGIA DE SOLUÇÕES DE ESPUMA SEMIRRÍGIDA DE POLIESTIRENO DISSOLVIDA EM D-LIMONENO
}

\author{
R.F. CELLA ${ }^{1}$; P.F. OLIVEIRA ${ }^{1}$; R.A.F. MACHADOํㄱ A. BOLZAN ${ }^{1}$ \\ ${ }^{1}$ Universidade Federal de Santa Catarina, Departamento de Engenharia Química e Engenharia de \\ Alimentos \\ E-mail para contato: rafaelfcella@yahoo.com.br
}

\begin{abstract}
RESUMO - O descarte do poliestireno expansível (EPS), um tipo de espuma semirrígida de poliestireno, traz sérios impactos à coleta e disposição dos resíduos. A reciclagem de EPS pós-consumo é considerada pouco atrativa, devido principalmente ao elevado custo de transporte e a contaminação dos materiais coletados. A reciclagem por dissolução propicia a redução do volume e possibilita a filtração para a retirada de impurezas. Uma das dificuldades na avaliação da viabilidade deste método é a determinação da concentração de EPS adequada ao processo de reciclagem. Na literatura são apresentados valores divergentes de solubilidade, variando entre $0,26 \mathrm{~g} / \mathrm{mL}$ e $0,56 \mathrm{~g} / \mathrm{mL}$. Propõe-se uma maneira alternativa de se avaliar a dissolução do EPS, através das concentrações críticas obtidas por experimentos de reologia. Neste trabalho foi medida a viscosidade a $25{ }^{\circ} \mathrm{C}$ de amostras de EPS dissolvidas em $d$-limoneno e foram determinados parâmetros reológicos, entre eles as regiões de concentração crítica. A transição da região muito diluída para a diluída variou entre 0,10 e $0,13 \mathrm{~g} / \mathrm{mL}$, e a concentração crítica que indica a transição da região diluída para a região onde são formados os emaranhamentos variou entre 0,24 e $0,27 \mathrm{~g} / \mathrm{mL}$. Foram obtidas soluções com concentração superior a $0,40 \mathrm{~g} / \mathrm{mL}$. Os valores determinados são importantes para a avaliação do processo de reciclagem, pois as concentrações críticas representam regiões onde há grande alteração no comportamento reológico.
\end{abstract}

\section{INTRODUÇÃO}

A utilização de espumas semirrígidas tem crescido a cada ano devido principalmente as propriedades térmicas, acústicas e mecânicas proporcionada aos materiais à custos relativamente baixos. O poliestireno (PS) é o polímero mais utilizado para esta finalidade, e suas espumas semirrígidas são conhecidas popularmente no Brasil por Isopor®. A reciclagem mecânica convencional do PS tem a desvantagem da degradação molecular do polímero causada pela oxidação e contaminação por outros materiais, o que resulta em um polímero reciclado de menor qualidade. Na literatura há trabalhos que citam a reciclagem de espuma semirrígida de poliestireno por dissolução em diferentes solventes, tais como acetona, 
tolueno, cloroforme, benzeno, xileno, tetrahidrofurano, pineno, mentol, cimeno e limoneno, entre outros. A dissolução das espumas semirrígidas de poliestireno em solventes orgânicos é uma alternativa para a reciclagem do polímero, reduzindo o volume transportado, possibilitando a filtração para limpeza e resultando em um produto de melhor qualidade quando comparado à reciclagem mecânica tradicionalmente utilizada para este material. No entanto, solventes derivados do petróleo não são favoráveis ao meio ambiente. Alguns solventes de fontes renováveis podem ser usados para dissolver as espumas semirrígidas de poliestireno, como limoneno, cimeno, terpineno, pineno, entre outros (Garcia et al., 2009a; Garcia et al., 2009b; Hattori et al., 2008). A reciclagem de poliestireno por dissolução usando o limoneno como solvente tem sido apontada como uma alternativa atrativa (Cella, 2012; Garcia et al., 2009a; Garcia et al., 2009b; Noguchi et al., 1998).

$\mathrm{Na}$ literatura são apresentados dados divergentes em relação a solubilidade de espumas semirrígidas de poliestireno em d-limoneno. Em experimentos a $50^{\circ} \mathrm{C}$, Garcia et al. (2009b) determinaram como $0,65 \mathrm{~g} / \mathrm{mL}$ o valor para solubilidade do poliestireno extrusado (XPS), um material semelhante ao EPS. Hattori et. al (2008), nesta mesma temperatura, determinaram a solubilidade do EPS em d-limoneno como 1,07 g/mL, enquanto Hattori et. al (2010) apresentou valores de solubilidade do EPS entre $1,53 \mathrm{~g} / \mathrm{ml}$ a $1,61 \mathrm{~g} / \mathrm{mL}$. Em ensaios a $25^{\circ} \mathrm{C}$, Garcia et al. (2009b) determinaram como $0,26 \mathrm{~g} / \mathrm{mL}$ o valor para solubilidade do XPS em d-limoneno. Em estudos de dissolução de EPS em d-limoneno a $25^{\circ} \mathrm{C}$, Cella (2012) relatou concentrações de até $0,59 \mathrm{~g} / \mathrm{mL}$, e Noguchi et. al (1998) determinaram a solubilidade do EPS em d-limoneno como aproximadamente $0,56 \mathrm{~g} / \mathrm{mL}$. A principal explicação para a grande diferença dos valores apresentados entre os diferentes autores é a utilização de alguma força para imergir as espumas semirrígidas no solvente.

No processo de reciclagem de polímeros por dissolução proposto por Papaspyrides et. al (1994) os valores máximos de concentração adequados as condições experimentais foram determinados empiricamente pelo comportamento reológico das soluções poliméricas. Estudos de Kampouris et. al (1987) e Kampouris et. al (1988), sobre reciclagem por dissolução-precipitação de espumas semirrígidas de poliestireno em solventes orgânicos, recomendam concentrações máximas de $0,30 \mathrm{~g} / \mathrm{mL}$ utilizando o MEK como solvente, 0,40 $\mathrm{g} / \mathrm{mL}$ utilizando o benzeno e $0,50 \mathrm{~g} / \mathrm{mL}$ com o tolueno. No processo de reciclagem de EPS por dissolução em d-limoneno e posterior secagem em secador de tambor, Cella (2012) relatou ser necessário concentrações superiores a $0,35 \mathrm{~g} / \mathrm{mL}$ para garantir a adequada aderência à superfície do tambor aquecido, sendo utilizadas concentrações de até $0,50 \mathrm{~g} / \mathrm{mL}$.

O objetivo deste trabalho é a determinação de concentrações que contribuam na avaliação de processos de reciclagem de espumas semirrígidas de poliestireno através de estudo do comportamento reológico da dissolução de EPS em d-limoneno.

\section{MATERIAIS E MÉTODOS}

\subsection{Preparação das amostras}

Foram utilizadas amostras de EPS provenientes de uma indústria recicladora. As amostras foram limpas antes dos experimentos e mantidas em banho termostático a temperatura de $25^{\circ} \mathrm{C}$ até a estabilização da temperatura, sendo então realizadas as medições 
de viscosidade. A concentração foi avaliada em massa de polímero por volume de solvente $(\mathrm{g} / \mathrm{mL})$.

\subsection{Medição da viscosidade das soluções muito diluídas}

A medição da viscosidade das soluções muito diluídas foi realizada com um viscosímetro capilar tipo Ubbelohde. As constantes do viscosímetro foram determinadas com água destilada a temperatura de $25{ }^{\circ} \mathrm{C}$. As amostras, com concentração entre $0,008 \mathrm{~g} / \mathrm{mL}$ e $0,042 \mathrm{~g} / \mathrm{mL}$ tiveram seus tempos de queda medidos por três vezes para cada uma das amostras, visando minimizar os erros experimentais.

\subsection{Determinação do coeficiente de Huggins e da viscosidade intrínseca}

$\mathrm{O}$ coeficiente de Huggins $\mathrm{k}_{\mathrm{H}}$ é um parâmetro adimensional dependente da temperatura que relaciona as interações entre polímero e solvente. É um coeficiente determinado em soluções muito diluídas (Fried, 1995), onde as soluções poliméricas tem um comportamento semelhante a um fluido newtoniado (Mello et al., 2006). A viscosidade relativa $\eta_{i}$, definida pela Equação 1, é relacionada com o coeficiente de Huggins através da Equação 2, onde $\eta$ é a viscosidade, $\eta_{0}$ a viscosidade do solvente puro, $c$ é a concentração do polímero na solução e [ $\eta]$ é a viscosidade intrínseca. A razão entre $\eta_{i}$ e $c$ é chamada de número de viscosidade ou viscosidade reduzida (Fried, 1995).

$$
\begin{aligned}
& \eta_{\mathrm{i}}=\frac{\left(\eta-\eta_{0}\right)}{\eta_{0}} \\
& \frac{\eta_{\mathrm{i}}}{c}=[\eta]+k_{H} \cdot[\eta]^{2} \cdot c
\end{aligned}
$$

Quanto menor o coeficiente de Huggins, mais solvatada a macromolécula do polímero se encontra (Mello et al., 2006). A viscosidade intrínseca é relacionada com a massa molar viscosimétrica do polímero (Fried, 1995).

\subsection{Medição da viscosidade aparente das soluções}

Soluções com concentração entre $0,14 \mathrm{~g} / \mathrm{mL}$ e $0,41 \mathrm{~g} / \mathrm{mL}$ tiveram suas viscosidades medidas com um viscosímetro digital de Krebs, com rotação constante de 200 rpm, fornecido pela empresa Sheen, modelo 480.

\subsection{Determinação das regiões de concentração critica}

As concentrações críticas são determinadas pelo gráfico do número de viscosidade, através da intersecção das retas que representam os períodos de crescimento linear do número de viscosidade (Fried, 1995). As concentrações críticas $c^{*}$ e $c^{* *}$ estão relacionadas a aumentos bruscos da viscosidade relativa com o aumento da concentração do polímero. A Figura 1 mostra cinco regiões possíveis para a solução polimérica, mostrando a transição das regiões muito diluídas para regiões diluídas $\left(c<c^{* *}\right)$, onde as moléculas dos polímeros estão isoladas (e o comportamento reológico é semelhante a um fluido newtoniado), até as regiões de média concentração $\left(c>c^{*}\right)$, onde os emaranhamentos estão presentes e há grande aumento no incremento de viscosidade com o aumento da concentração (Fried, 1995). 


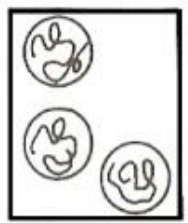

$\mathrm{C}<\mathrm{C}^{\star *}$

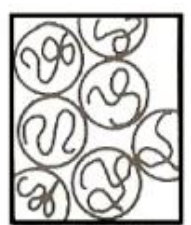

$\mathrm{C}=\mathrm{C}^{* x}$

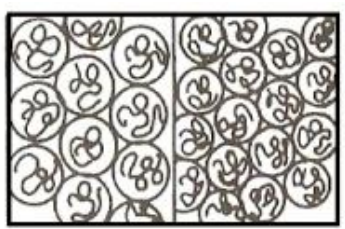

$\mathrm{C}^{* *}<\mathrm{C}<\mathrm{C}^{*}$

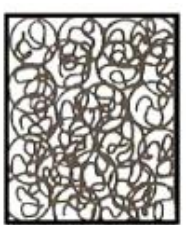

$\mathrm{C}=\mathrm{C}^{*}$

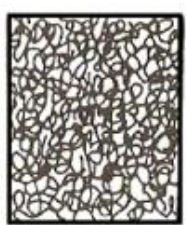

$C>C^{*}$

Figura 1 - Regiões de concentrações críticas.

\section{RESULTADOS E DISCUSSÕES}

\subsection{Viscosidade das soluções muito diluídas}

A Figura 2 mostra as viscosidades absolutas medidas para as soluções muito diluídas a temperatura de $25^{\circ} \mathrm{C}$.

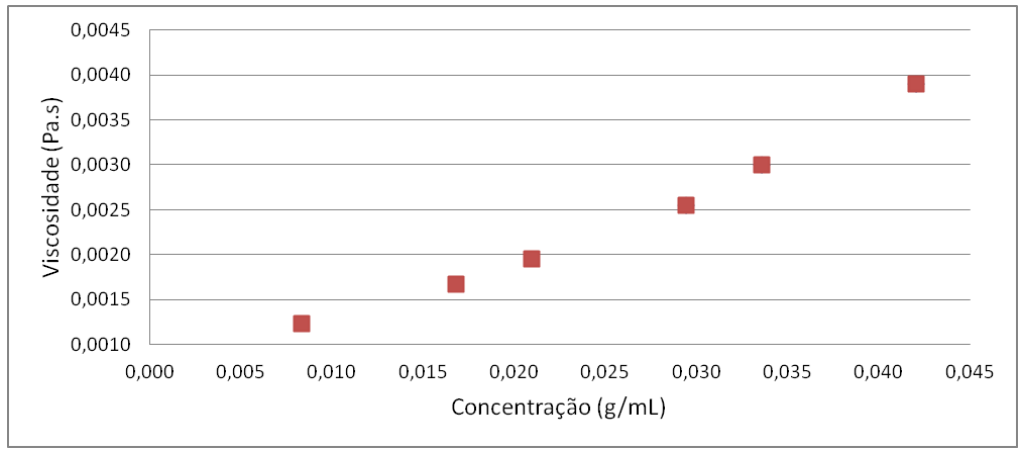

Figura 2 - Viscosidade absoluta das soluções muito diluídas.

\subsection{Coeficiente de Huggins e viscosidade intrínseca}

A Figura 3 mostra o número de viscosidade para as soluções muito diluídas e as retas originadas pela linearização. $O$ coeficiente de Huggins e a viscosidade intrínseca são determinados pelas equações resultantes da linearização, conforme a Equação 2. O valor da viscosidade intrínseca obtido foi de $42,2 \mathrm{~mL} / \mathrm{g}$ e o valor da constante de Huggins foi de 0,54 a $25^{\circ} \mathrm{C}$.

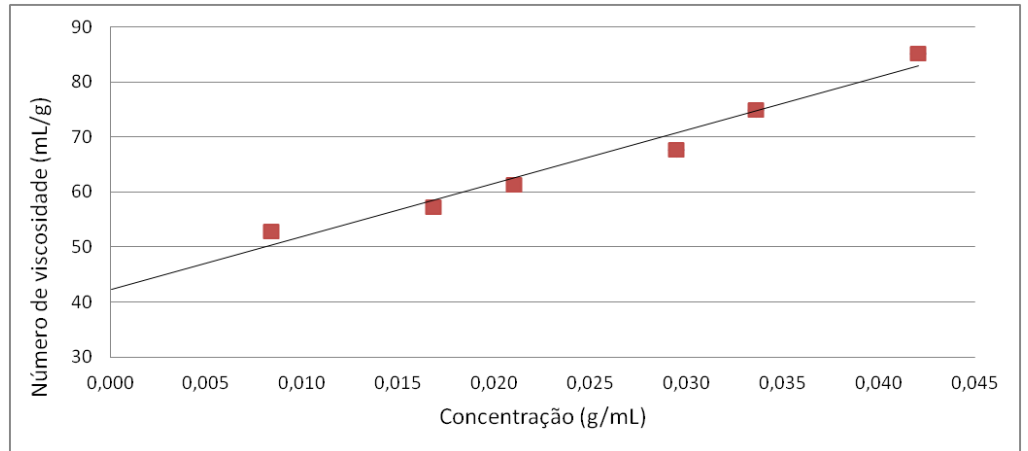

Figura 3 - Número de viscosidade para soluções muito diluídas. 


\subsection{Viscosidade aparente das soluções}

A Figura 4 apresenta os resultados de viscosidade aparente para três amostras de soluções de EPS dissolvido em d-limoneno a $25^{\circ} \mathrm{C}$.

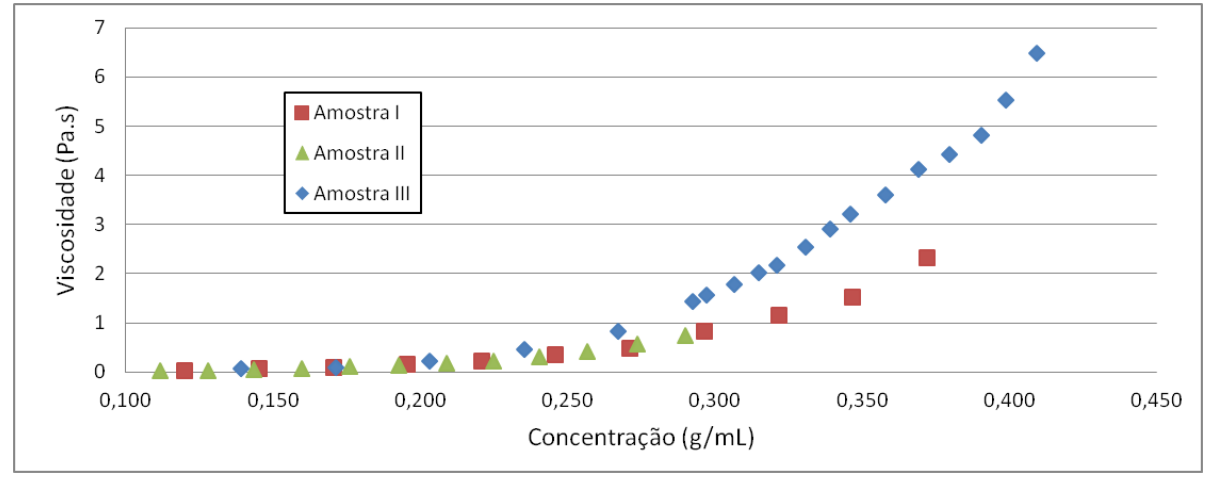

Figura 4 - Viscosidade aparente.

Em estudo comparando a viscosidade de soluções de dissolvido em cinco tipos de solventes orgânicos, Kampouris (1987) utilizou como parâmetro o logaritmo da viscosidade para a viscosidade de EPS em solventes orgânicos, em concentrações entre $0,10 \mathrm{~g} / \mathrm{mL}$ e 0,40 $\mathrm{g} / \mathrm{mL}$. A Figura 5 apresenta o gráfico do logaritmo da viscosidade com a concentração das soluções de EPS dissolvido, com valores entre $0,11 \mathrm{~g} / \mathrm{mL}$ e $0,41 \mathrm{~g} / \mathrm{mL}$. Não é notada uma relação linear em todas as regiões de concentração, mas sim um comportamento constante em determinadas regiões. A Figura 6 apresenta o gráfico do número de viscosidade.

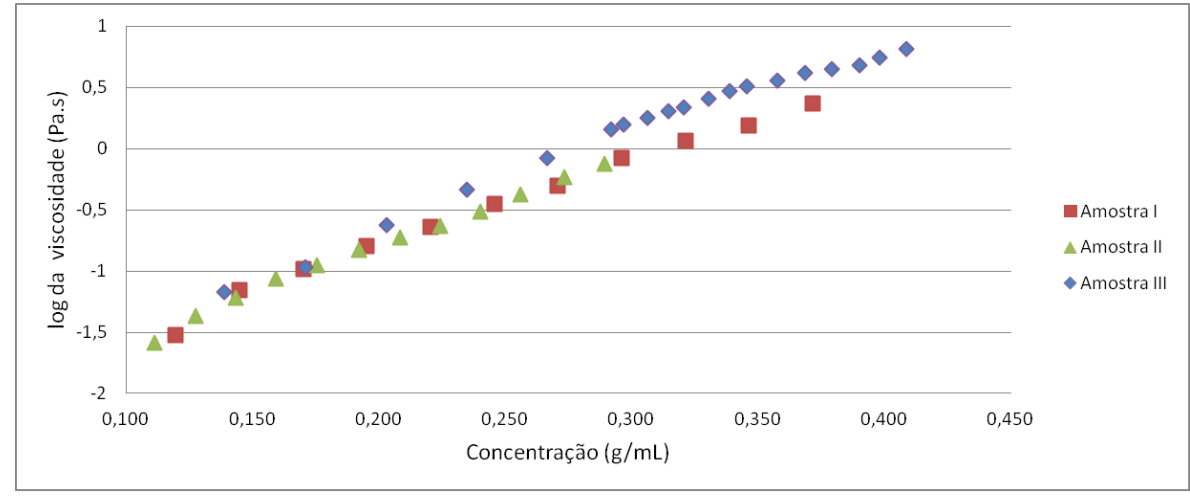

Figura 5 - Logaritmo da viscosidade.

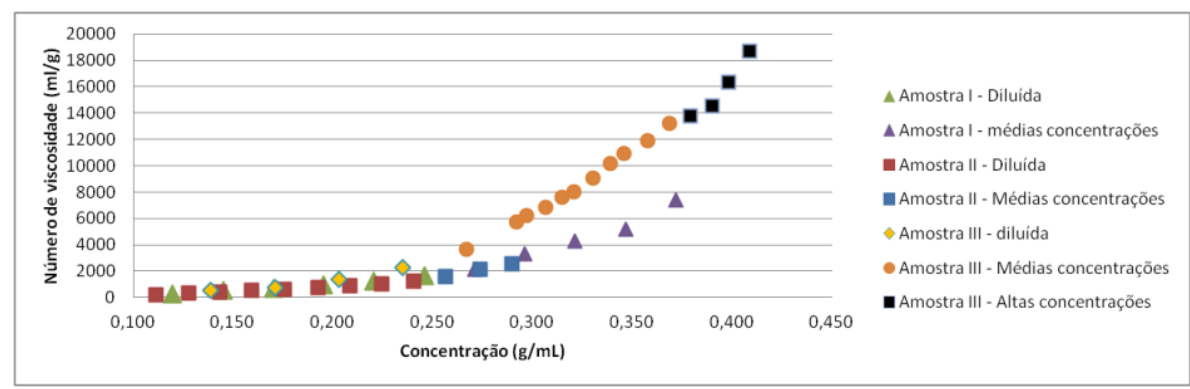

Figura 6 - Número de viscosidade. 


\subsection{Concentrações críticas}

A Figura 7, a Figura 8 e a Figura 9 apresentam os três períodos de crescimento linear do número de viscosidade e suas retas obtidas pela linearização para as três amostras. As intersecções são as concentrações críticas.

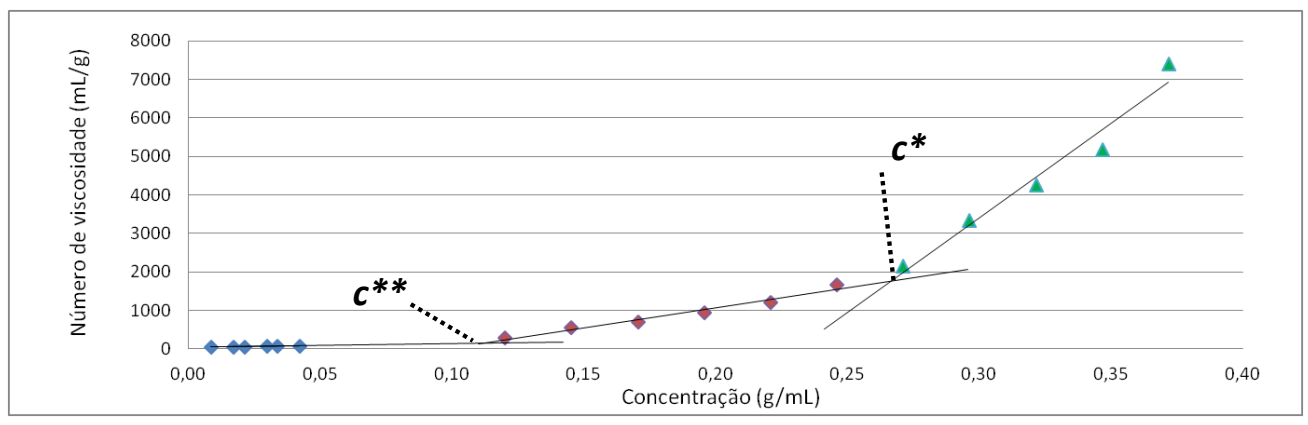

Figura 7 - Concentrações críticas para a amostra I a $25^{\circ} \mathrm{C}$.

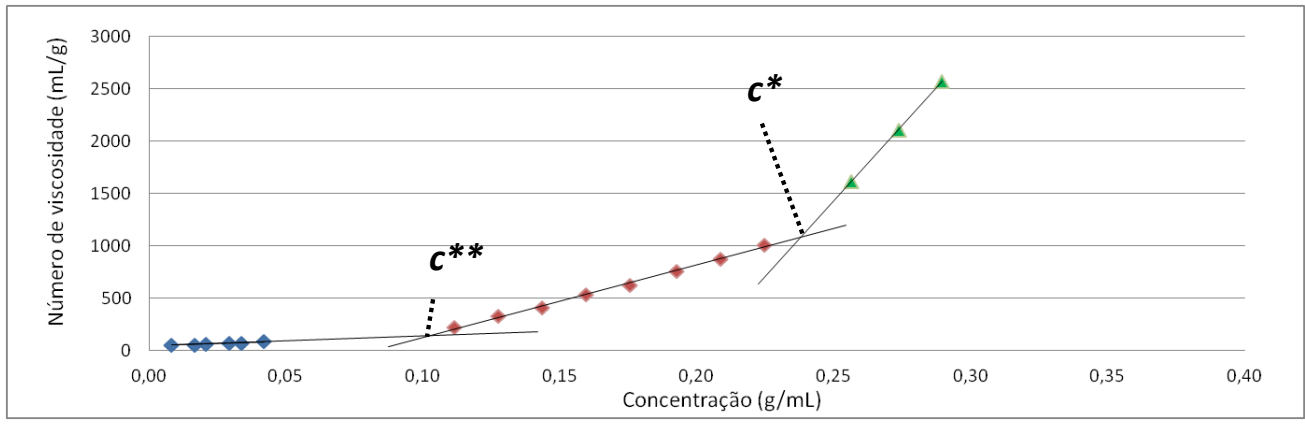

Figura 8 - Concentrações críticas para a amostra II a $25^{\circ} \mathrm{C}$.

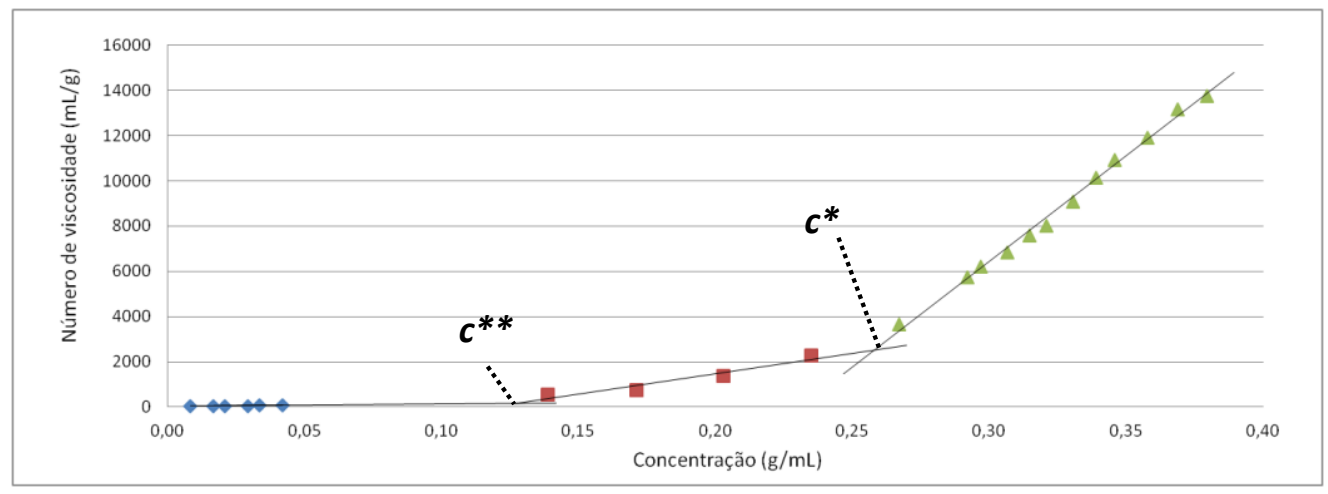

Figura 9 - Concentrações críticas para a amostra III a $25^{\circ} \mathrm{C}$.

Os resultados da determinação das concentrações críticas são apresentados na Tabela 2. 
Tabela 2 - Resultados das concentrações críticas

\begin{tabular}{|c|c|c|}
\hline Amostra & $\boldsymbol{c}^{* *}(\mathbf{g} / \mathbf{m L})$ & $\boldsymbol{c}^{*}(\mathbf{g} / \mathbf{m L})$ \\
\hline Amostra I & 0,11 & 0,27 \\
\hline Amostra II & 0,10 & 0,24 \\
\hline Amostra III & 0,13 & 0,26 \\
\hline
\end{tabular}

Através da variação da viscosidade das soluções poliméricas, sugere-se que a dissolução de espuma semirrígida de poliestireno seja divida em quatro regiões de diferentes concentrações:

- Muito diluída: concentração inferior a $\mathrm{c}^{* *}$. Há um aumento linear da viscosidade com o aumento da concentração, e esta região é importante para determinação de parâmetros reológicos devido a um comportamento similar a um fluido newtoniado (Mello et. al, 2006).

- Diluída: concentração superior a $\mathrm{c}^{* *}$ e inferior a $\mathrm{c}^{*}$. Há um incremento no aumento da viscosidade com o aumento da concentração. É uma região onde o processo de reciclagem tende a ser mais simples, pois não há aderência excessiva com as superfícies em que entra em contato (Kampouris et. al, 1988).

- Médias concentrações: concentração superior a c*. Região onde são formados os emaranhamentos de polímero. A concentração c* é próxima de valores publicados na literatura por alguns autores como valor de solubilidade, como os valores publicados por Garcia et. al (2009a) e Garcia et. al (2009b) para espumas semirrígidas. Nesta região há um crescimento exponencial da viscosidade com o aumento da concentração. Em alguns processos industriais de reciclagem pode ser de interesse pela alta aderência (quando esta é necessária, como em processos de secagem por secador de tambor), mas de maneira geral a alta aderência aumenta a possibilidade de perdas durante o processamento.

- Altas concentrações: concentrações onde a solução apresenta-se extremamente viscosa, o que limita a dissolução de mais material, e a velocidade de dissolução é bastante reduzida. Para a dissolução das amostras de EPS em d-limoneno deste trabalho, esta região inicia em concentrações entre $0,36 \mathrm{~g} / \mathrm{mL}$ e $0,39 \mathrm{~g} / \mathrm{mL}$. Nesta região há um crescimento exponencial do número de viscosidade com o aumento da concentração. A concentração da solução polimérica pode atingir valores superiores a $0,55 \mathrm{~g} / \mathrm{mL}$ a temperatura de $25^{\circ} \mathrm{C}$ (Cella, 2012; Noguchi et. al, 1998).

\section{CONCLUSÃO}

Os valores de concentrações críticas são importantes para a avaliação do processo de reciclagem de espumas semirrígidas de poliestireno por dissolução, pois as concentrações críticas representam regiões onde há grande alteração no comportamento reológico. 


\section{REFÊNCIAS BIBLIOGRÁFICAS}

CELLA, Rafael Ferraz. Reciclagem de Espumas Semi-rígidas de Poliestireno pela Dissolução em Terpenos e Secagem em Secador de Tambor. Dissertação (mestrado). Programa de Pós-Graduação em Engenharia Química - Universidade Federal de Santa Catarina, Florianópolis, 2012.

FRIED, Joel R. Polymer Science and Technology. New Jersey, USA: Prentice Hall PTR, 1995.

GARCÍA, M.T.; DUQUE, G.; GRACIA, I.; LUCAS, A.; RODRÍGUEZ, J.F. Recycling extruded polystyrene by dissolution with suitable solvents. Journal Of Material Cycles And Waste Management. p. 2-5. 2009a.

GARCÍA, M.T.; GRACIA, I.; DUQUE, G.; LUCAS, A.; RODRÍGUEZ, J.F. Study of the solubility and stability of polystyrene wastes in a dissolution recycling process. Waste Management. p. 1814-1818. 2009b.

HATTORI, K.; NAITO, S.; YAMAUCHI, K.; NAKATANI, H.; YOSHIDA, T.; SAITO, S.; AOYAMA, M. Solubilization of Polystyrene into Monoterpenes. Advances in Polymer Technology, v. 27, p. 35-39. 2008.

KAMPOURIS, E. M.; PAPASPYRIDES, C. D.; LEKAKOU, C. N. A model recovery process for scrap polystyrene foam by means of solvent systems. Conservation \& Recycling, v.10, f. 4, p. 315-319, 1987.

KAMPOURIS, E. M.; PAPASPYRIDES, C. D.; LEKAKOU, C. N. A model process for the solvent recycling of polystyrene. Polymer Engineering \& Science, v.28, f.8, p. 534537, 1988.

MELlO, I.L.; COUTINHO, F.M.B.; DELPECH, M.C.; ALBINO, F.F.M.; SANTOS, S.M. Polibutadieno Alto-cis: Estudo Viscosimétrico em Tolueno e Ciclo-hexano. Polímeros: Ciência e Tecnologia. V. 16, n. 1, p. 53-60. 2006.

NOGUCHI T.; MIYASHITA M., INAGAKI Y., WATANABE H. A new recycling system for expanded polystyrene using a natural solvent. Part 1. A new recycling technique. Packaging Technology and Science, v. 11, p. 19-27. 1998.

PAPASPYRIDES, C.D.; POULAKIS, J.G.; VARELIDES, P.C. A model recycling process for low density polyethylene. Resources, Conservation and Recycling, v.12, p. 177184, 1994. 